\title{
Earth gravity from space or how attractive is our planet?
}

\author{
$\therefore$. Reiner Rummel - rummel@bvitum.de - DOI: 10.1051/epn/2013103 \\ I Astronomical and Physical Geodesy - Technische Universität München -80 290 München - Germany
}

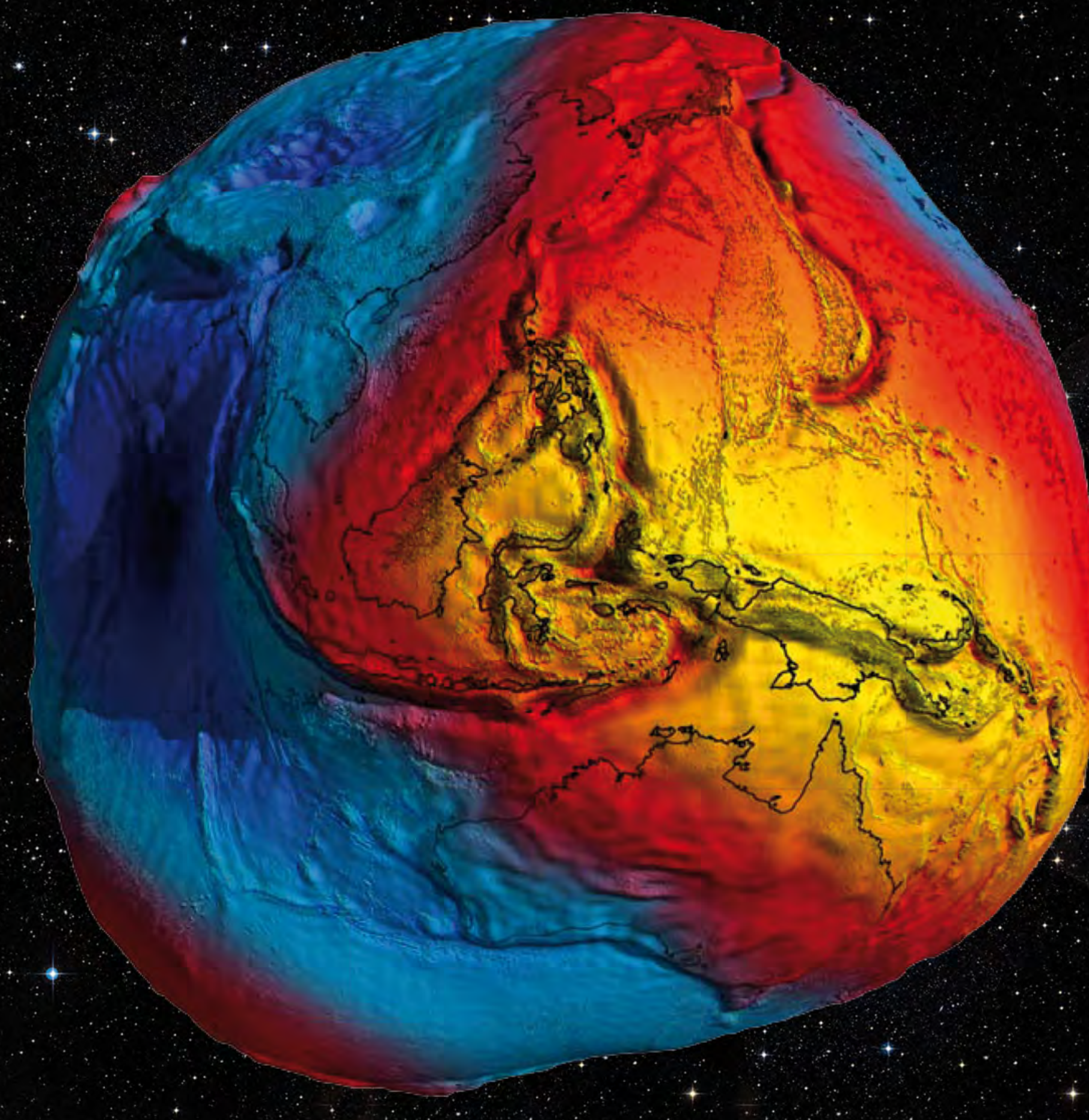




\section{In March 2011 the European Space Agency (ESA) released an impressive new picture of the figure of the earth. It was based on the first science data from the satellite GOCE. It shows the geoid with its undulations highly exaggerated, which makes the Earth look almost like a potato, but very attractive. The picture received great attention from the media and even made it to the front page of many leading international newspapers.}

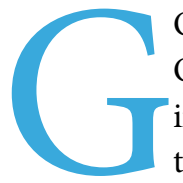

OCE (acronym for Gravity and steady-state Ocean Circulation Explorer) was launched in March 2009. It is still in orbit. The end of the mission is projected for the second half of 2013, when the spacecraft will have used up most of the Xenon of its ion propulsion system, which is needed to keep it in an extremely low orbit. Its primary goal is the global determination of the geoid and of gravity with an accuracy of one part per million (1 ppm). To achieve these objectives the principle of gravitational gradiometry is applied, the first time ever in a spacecraft. The gradiometer instrument consists of three pairs of ultra-precise accelerometers, and the satellite is essentially a laboratory built around the gradiometer. In order to enhance the gravitational signal the orbit altitude is chosen very low, only $255 \mathrm{~km}$. Despite the high accuracy of the gradiometer instrument, data analysis can still be based on Newton's universal law of gravitation.

Gravity is the combined effect of 'true' gravitation and the centrifugal acceleration due to earth rotation. In first approximation, for a spherical earth it is a constant, the well-known $9.8 \mathrm{~m} / \mathrm{s}^{2}$. The deviations from this value are small. The largest, of the order of $1 / 300$, is due to the combined effect of centrifugal acceleration and of the Earth's oblateness which is essentially a result of Earth rotation. Newton was the first to use gravity measurements as a confirmation of his theory about equilibrium figures and the earth's flattening. For this purpose he considered 'seconds pendulums' employed for astronomical measurements, and analysed the adjustments applied to their length at stations at various latitudes.

\section{Density variations}

Gravity differences due to topography and density variations in the earth's interior are even smaller; they are less than $10^{-4}$. They are caused by phenomena ranging from large-scale density variations due to tectonic processes and mantle convection, to small-scale effects such as cavities in the underground or density jumps in soil or rock material. For this latter reason, terrestrial gravimetry belongs to the standard methods of exploration geophysics. Temporal variations of gravity are less than $1 \mathrm{ppm}$. The largest contribution is due to the tidal effect of sun, moon and planets on the oceans and the deformable earth. Smaller contributions are caused, e.g., by changing groundwater levels and variations of atmospheric pressure. Some contributions and their size are listed in Table 1.

The traditional measurement techniques are absolute gravimetry, employing rather sophisticated free-fall apparatus [1], and relative gravimetry with spring gravimeters. Temporal variations are measured most accurately by superconducting gravimeters, stationary and under laboratory conditions. They are also relative gravimeters. Their relative sensitivity is as high as about $10^{-12}$.This approach employs a superconducting sphere which is kept levitated by a magnetic field of superconducting, persistent current coils [2]. However, even more than fifty years of terrestrial measurement activity worldwide resulted only in a rather incomplete and incoherent situation. Only in some highly developed countries dense and accurate gravity networks exist. In a much larger part of our world gravimetric measurements are either missing or they are sparse, inhomogeneous and inaccurate.

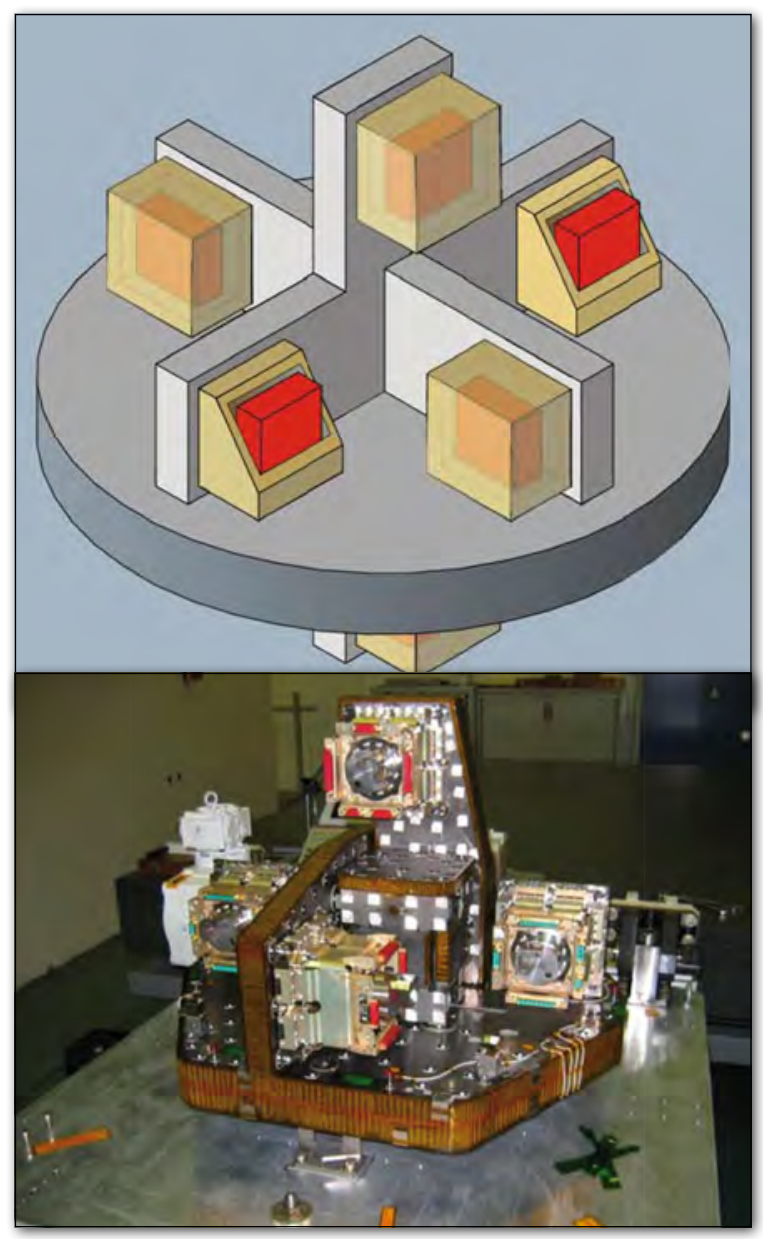

4 FIG. 1: The GOCE geoid "potato". The geoid undulations are highly exaggerated. Their maximum values, expressed relative to a best fitting global ellipsoid, are -100 meters South of India and +80 meters around New Guinea (courtesy: ESA)

FIG. 2: The GOCE gravitational gradiometer consists of three perpendicular one-axis gradiometers. Each of them comprises a rigid bar with two accelerometers at its ends. The accelerometers are the gravity signal sensors. In each of them a test mass ( 4 $\mathrm{cm} \times 4 \mathrm{~cm} \times 1 \mathrm{~cm}$ ) is kept levitated by an electrostatic feedback system. The applied current is proportional to the gravitational acceleration signal.

Upper panel: principle of the gradiometer with its six cubic test masses (in red).

Lower panel: the actual GOCE gradiometer instrument (courtesy: ESA), 


\section{BOX 1}

The complete gravitational tensor consists of the nine second derivatives $V_{i j}=\partial^{2} V / \partial x^{i} \partial x^{j}$ of the gravitational potential at the location of the satellite. Due to its symmetry only six elements remain. GOCE measures four of them with high precision, namely $V_{x x}, V_{y y} V_{z z}$ and $V_{x z}$ while $V_{x y}$ and $V_{y z}$ are measured much less accurately. Geometrically, the tensor elements represent the local curvature structure of the field, i.e., the curvature of the equipotential surfaces and plumb lines. Equivalently, the accelerations may be interpreted as measurement of the tidal field generated by the attraction of the Earth inside the GOCE satellite, cf. [4].
L 1

\section{In order to recover the small variations of the earth's field, the relative precision has to be 1 ppm 7 ?}

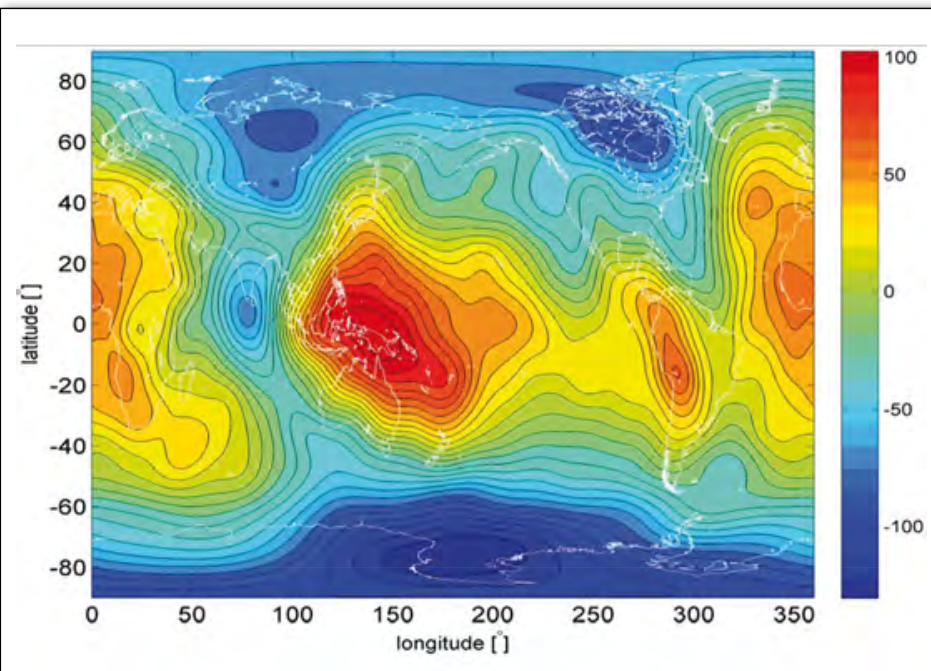

\section{Satellites}

With satellites the earth's gravitational field can be measured globally, homogeneously, fast and much more conveniently. The tracking of any earth-orbiting satellite from observatories on ground may be regarded as measurement of the free fall of a test mass, the satellite itself, in the gravitational field of the earth. The orbit trajectory is a Keplerian ellipse; it is precessing in space due to the earths flattening, and modulated by the gravitational signal caused by field variations originating from the earth, and - to a much lesser extent - from the sun, moon and planets. The gravitational signal is like a successive series of tiny accelerations and decelerations along the orbit. Already in 1958, with data from Sputnik-1 and Sputnik-2, the Earth's flattening could be determined from orbit analysis much more accurately than from the preceding 150 years of classical triangulations all around the world. With the advent of the Global Positioning System (GPS) a further break-through happened. Now the orbit of any satellite in a low orbit can be followed from the constellation of GPS-satellites in much higher orbit uninterruptedly, in three dimensions and with $\mathrm{cm}$ precision. However, while this approach proved rather successful, being global, uniform and fast, an intrinsic problem remained. It is the limitation of spatial resolution due to the large distance of satellites from the Earth. The strength of the field is quickly decreasing with increasing distance from the earth as expressed by Newton's "inverse squared distance law". v FIG. 3: GOCE sensor system with the gradiometer instrument in its center,
a geodetic GPS-receiver, three star trackers, two ion thrusters at its rear end,
magnetic torquers and cold gas thrusters for calibration (courtesy: ESA)

4 FIG. 4: Global map of geoid heights (in meters) relative to an ellipsoidal figure in hydrostatic equilibrium

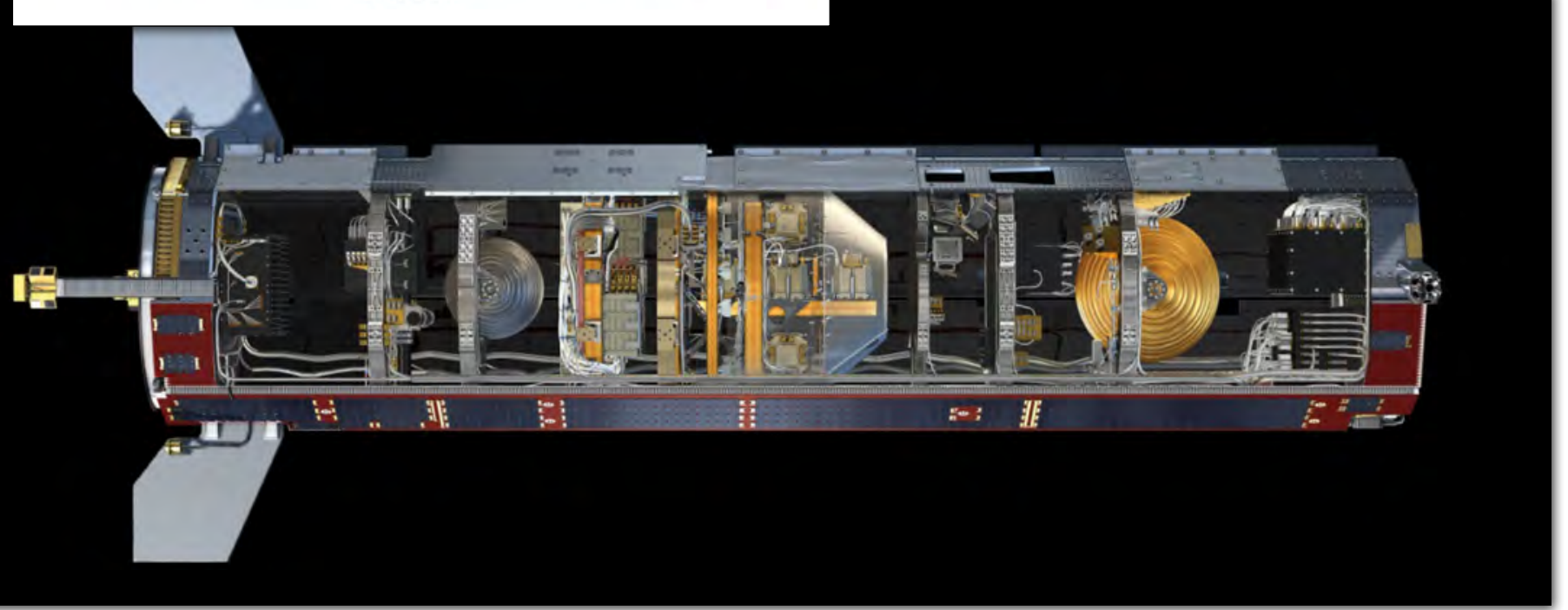




\begin{tabular}{|c|c|}
\hline \multicolumn{2}{|c|}{ EARTH GRAVITY AND ITS CONTRIBUTIONS } \\
\hline Size of effect relative to $\mathrm{g}\left[\right.$ in $\left.\mathrm{m} / \mathrm{s}^{2}\right]$ & The various contibutions \\
\hline $10^{0}$ & Earth as a homogeneous sphere \\
\hline $10^{-3}$ & Oblateness and centrifugal acceleration \\
\hline $10^{-4}$ & Mountains, valleys, ocean ridges, subduction zones \\
\hline $10^{-5}$ & Density variations in lithosphere and upper mantle \\
\hline $10^{-6}$ & Sediments, salt domes, ores \\
\hline $10^{-7}$ & Temporal variations due to solid earth and ocean tides \\
\hline $10^{-8}$ & Loading effects, atmospheric pressure, groundwater variations \\
\hline $10^{-9}$ & Pole tide, sea level \\
\hline
\end{tabular}

$\triangle$ TABLE 1:

Earth gravity and its contributions

\section{Gravitational gradiometry}

For this reason GOCE is applying the principle of gravitational gradiometry. Gravitational gradiometry is designed to counteract the signal attenuation at orbit altitude. Instead of measuring the "free fall" of a single satellite, gravitational gradiometry is the measurement of the relative motion between several test masses. In the case of GOCE six test masses are used. The gradiometer consists of three orthogonal single-axis gradiometers, each $50 \mathrm{~cm}$ long, with their common midpoint at the satellite's centre of mass. The $\mathrm{x}$-axis is parallel to the satellite body of GOCE, roughly in flight direction, the $\mathrm{z}$-axis is pointing towards the Earth and the $y$-axis is perpendicular to the former two, approximately orthogonal to the plane of the orbit. Each singleaxis gradiometer has two very precise accelerometers at its end points. These contain a $4 \mathrm{~cm} \times 4 \mathrm{~cm} \times 1 \mathrm{~cm}$ cubic test mass, made of Rhodium-Platinum. Their weight is 320 gram. Each test mass is kept levitated inside its housing without touching its walls, suspended by an electrostatic feed-back system [3].If one of these accelerometers would be placed exactly at the satellite's centre of mass, it would sense zero- $g$ : The gravitational attraction of the Earth on the accelerometer's test mass would be identical to that on the satellite. At $25 \mathrm{~cm}$ distance from the satellite's centre the accelerometers are operating in a "micro-g"-environment. They sense the tiny gravitational acceleration difference of the Earth's field between the location of the test mass and that of the satellite's centre of mass, just $25 \mathrm{~cm}$ above, below, in front, behind, to the left or to the right of it. The signal size is less than $10^{-6}$ of $g$. In order to recover the small variations of the earth's field, caused by mountains and valleys, ocean ridges, subduction zones or mantle convection, the relative precision has to be $1 \mathrm{ppm}$. See also Box 2 . This implies that the required sensitivity of the accelerometers is of the order of $10^{-12} \mathrm{~m} / \mathrm{s}^{2}$. The gravitational gradients are then the differences of the measured accelerations in $\mathrm{x}, \mathrm{y}$ and $\mathrm{z}$ direction along each of the three gradiometer arms. The gradiometer instrument is shown in Figure 2.

Since the gradiometer instrument is rigidly mounted in the spacecraft and the satellite is Earth-pointing it performs one full rotation with each orbit revolution. Essentially this

is a rotation about the out-of-orbit-plane y-axis in inertial space. Consequently, the gravitational signal is superimposed by centrifugal and angular accelerations. They have to be reconstructed and eliminated. While the gravitational and centrifugal part of the $3 \times 3$ matrix formed by the nine

\section{BOX 2}

We see a "satellite" with four freely floating test masses in its interior, in orbit around the earth. If one of these test masses would be placed exactly at the centre of mass of the satellite it would remain there. Seen relative from the satellite, it feels "zero-g". Seen from the outside, the

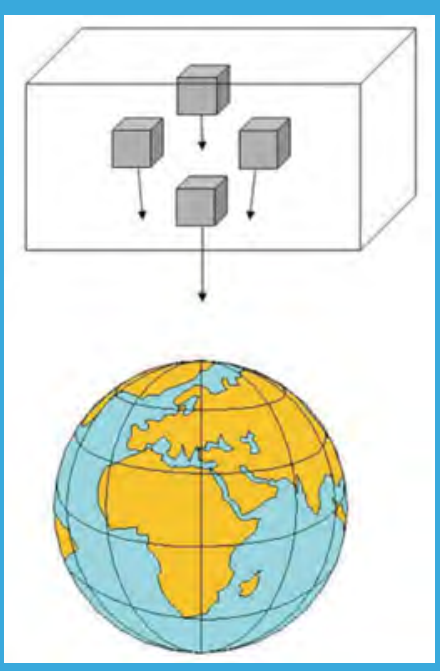
gravitational force of the earth on the satellite is identical in magnitude and direction to that on the test mass. There is no acceleration difference. If the test masses are placed slightly "higher" or "lower" or in front or behind the satellite's centre of mass, as shown in the figure, they are attracted by the earth slightly more or less or in a different direction compared to the satellite, as indicated by the arrows in the figure. So the test masses are in a"micro$g^{\prime \prime}$-environment. There is a tiny difference between earth gravitation on the satellite and on each of the four test cubes. At a distance of one meter from the satellite centre this difference is of the order of one millionth of $g$.

In the case of the GOCE gradiometer, the test masses are kept at a fixed position using an electrostatic feedback system. The gradients are derived from the measured acceleration differences. - 
acceleration differences is symmetric, the angular accelerations part is skew-symmetric. It can therefore be identified. The angular accelerations are then combined with the observations of a set of three highly sensitive star trackers in order to reconstruct the angular rates [5]. In parallel to the gradiometric method, the gravity field is deduced (with much less spatial details) from the GOCE-orbits in a classical manner, as mentioned above. The orbits are reconstructed purely geometrically from the ranging of GOCE relative to the GPS constellation, i.e., without employing orbit mechanics. Regular comparisons with laser distance measurements from several ground stations confirm their centimetre accuracy.

\section{Atmospheric drag compensation}

A further novelty of GOCE is its extremely low orbit altitude of only $255 \mathrm{~km}$. Such low orbit requires continuous compensation of atmospheric drag. The drag effect on the spacecraft is measured by the complete set of accelerometers as common-mode signal and counteracted by a set of two proportional ion-thrusters. The orbit is sunsynchronous with an inclination of the orbit plane of 96.7 degrees relative to the equator plane. In 61 days GOCE covers the Earth with a dense net of ground tracks. After each of these orbit cycles the gradiometer is re-calibrated. This is done by randomly shaking the satellite with a set of cold-gas thrusters. Time varying gravitational effects coming from the satellite itself are minimized. For this reason the solar panels are rigidly mounted to the satellite body; the satellite structure is stiffened using carbon sandwich structures and the gradiometer instrument is operating under almost perfect thermal stability. The complete sensor system is shown in Figure 3. In summary, the gradiometer is operating in a laboratory environment, perfectly tailored to the needs of a gravitational experiment of this high standard. Since November 2009 GOCE is delivering science data [6], see also [7].

Figure 1 shows the global geoid as derived from the first six months of data. The bumps and dips shown there represent the deviations of the geoid from a best fitting ellipsoid; they range from $-100 \mathrm{~m}$ south of India to $+80 \mathrm{~m}$ around New Guinea and they are caused by large density contrasts deep in the Earth's mantle. The geoid is one of the level surfaces of the Earth's gravity field. It is the equipotential surface at mean sea level. Probably more revealing than figure 1 is a comparison of the geoid to an ellipsoidal figure in hydrostatic equilibrium, see Figure 4. It nicely emphasizes the imbalance between the major zones of upwelling hot mantle material, the so-called ring of fire, and those with colder material descending from the surface into the mantle. If one removes the long wavelength structure of this map, say scales longer than $1000 \mathrm{~km}$, the high correlation of the remaining geoid looks almost like an introduction into geodynamics. This is done in Figure 5. It emphasises all tectonic features of the lithosphere and upper mantle, such as subduction zones, ocean ridges, transform faults and zones of postglacial uplift.

This immediately suggests employing the GOCE geoid and gravity maps together with seismic tomography for a joint inversion, with the goal of improving global and regional Earth models. Several groups are currently working in this direction. First tests show that GOCE is producing much more consistent and accurate gravity and geoid maps of South America, Africa, Himalaya, South-East Asia and, very importantly, Antarctica [8]. It may also shed light on the open question of a suspected large meteorite impact in Wilkes Land, probably hidden under Antarctica's thick ice shield.

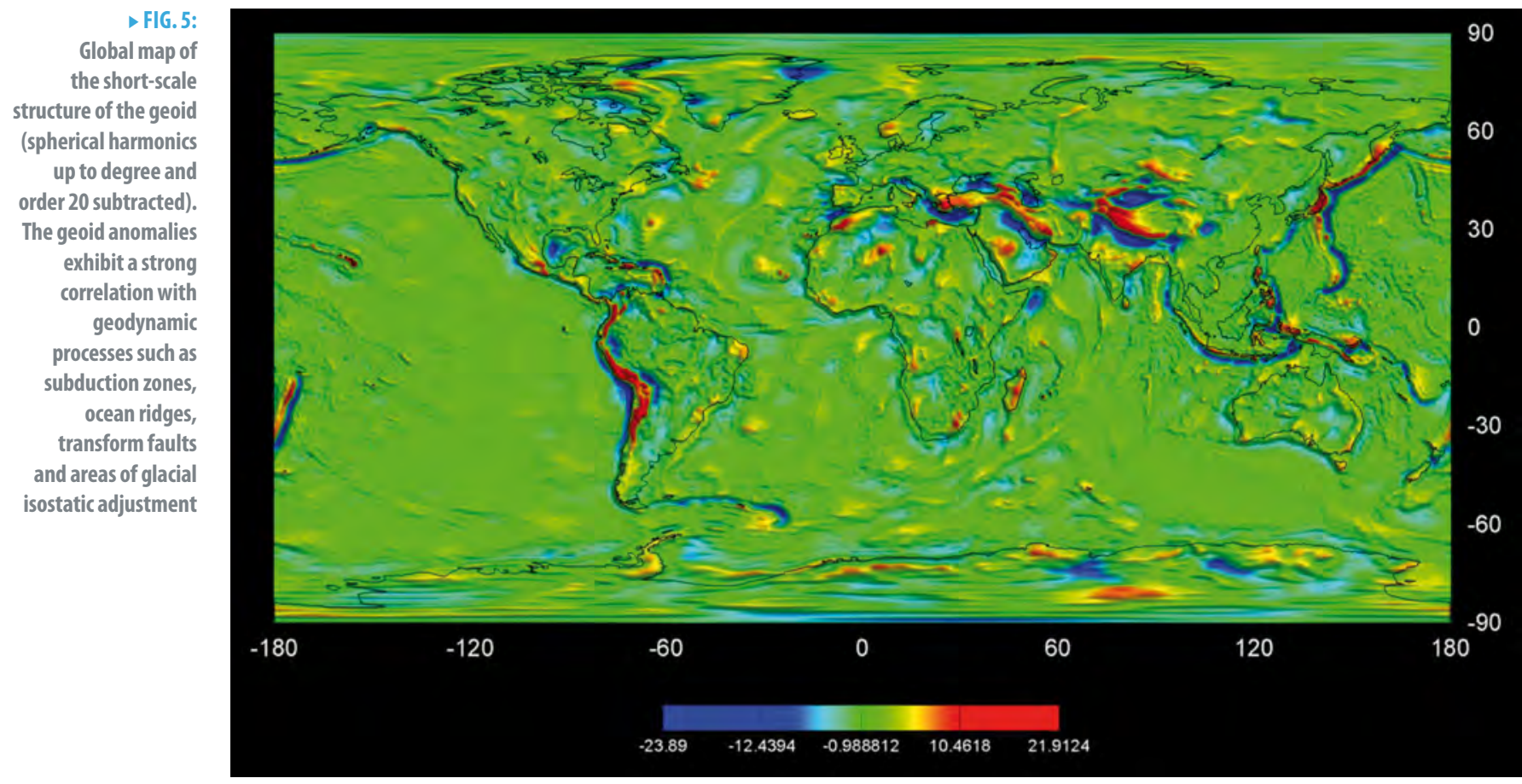




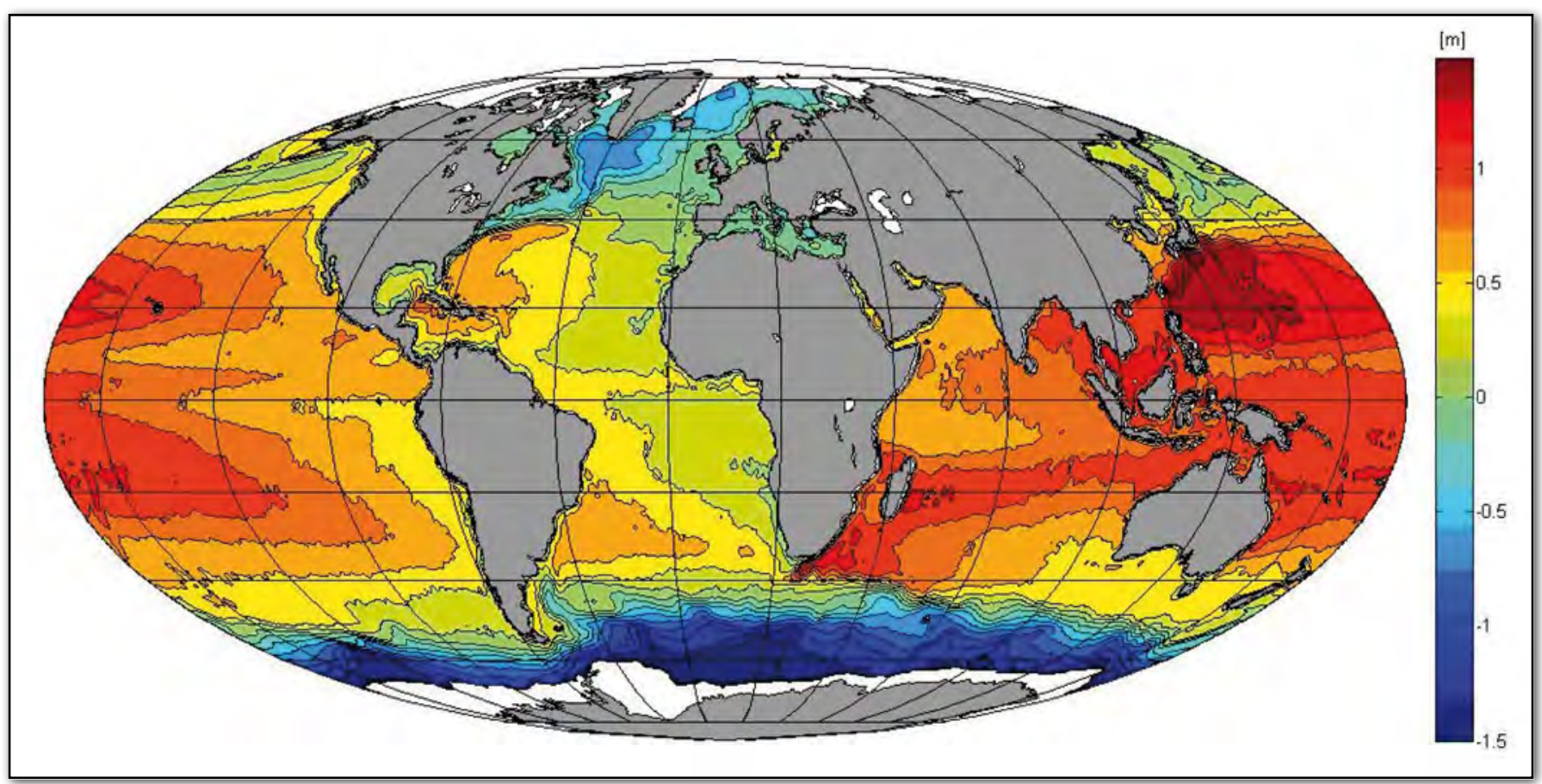

\section{Oceanography}

While in solid-earth physics the geoid serves as a measure of mass imbalance, its role in oceanography is that of the hypothetical ocean surface at rest. Without tides, winds and the effect of atmospheric pressure differences the ocean surface would coincide with the geoid. The deviation of the actual ocean surface from the geoid is therefore a measure of the strength of ocean circulation. Several very accurate models of the actual mean sea surface exist. They are derived from a 20-year long sequence of satellite radar altimetry missions. The difference of the two surfaces, the altimetric mean sea surface and the GOCE geoid, gives the mean dynamic ocean topography, representing the deviation of the actual sea surface from its hypothetical state-of-rest. The height variations are only of the order of 20 to $30 \mathrm{~cm}$, with maximum values up to 1 to 2 metres in the major ocean circulation systems such as the Gulf Stream, the Kuroshio Stream or the Antarctic Circumpolar Current, as shown in Figure 6. Ocean topography is maintained by the balance of the pressure differences induced by the dynamic topography and the Coriolis force on the water masses moving relative to the rotating earth. From the slopes of the mean dynamic ocean topography follows directly the strength of the ocean flow [9]. It is now for the first time, that the variations of the mean dynamic ocean topography are available, globally consistent, accurately and with such high spatial detail. They are derived from space without the aid of any oceanic in-situ data or models. It is important new input for improved numerical models of the ocean and for improved estimates of heat and mass transport in the oceans.

The GOCE geoid model is also needed for a unification of the national and regional height systems around the world. First trials with height data from North America,
Europe and Australia have revealed off-sets of close to one metre. A globally consistent height reference is needed for sea level studies, geo-information systems and the conversion of GPS-heights to physically meaningful heights above the geoid. GPS-heights alone are simply heights above a conventionally adopted reference ellipsoid.

All sensor systems of GOCE are functioning well. The expectation is that after re-processing and analysis of the full data set from November 2009 to summer 2012 all mission objectives will be met. Currently plans are discussed to use the last year of the mission (from autumn 2012 to autumn 2013), to descend the satellite to an even lower orbit, ultimately by $20 \mathrm{~km}$. This will further enhance the sensitivity and lead to a further improved spatial resolution and accuracy of the GOCE geoid and gravity field world map.

\section{References}

[1] J.E. Faller and I. Marson, Metrologia 25, 49 (1988).

[2] J.M. Goodkind, Rev. Sci. Instrum. 70, 4131 (1999).

[3] P. Touboul, Space Science Review 108, 393 (2003).

[4] H.C. Ohanian, R. Ruffini, Gravitation and Spacetime, $2^{\text {nd }}$ edition, Norton, (1994).

[5] R. Rummel, W. Yi, C. Stummer, Journal of Geodesy 85, 777 (2011).

[6] R. Floberghagen, M. Fehringer, D. Lamarre, D. Muzi, B. Frommknecht, C. Steiger, J. Piñeiro and A. da Costa, Journal of Geodesy 85, 749 (2011).

[7] Q. Schiermeier, Nature 467, 648 (2010).

[8] F. Ferraccioli, C.A. Finn, T.A. Jordan, R.E. Belt, L.M. Anderson and D. Damaske, Nature 479, 388 (2011).

[9] R.J. Bingham, P. Knudsen, O. Andersen and R. Pail, Geophys. Res. Lett. 38, L01606 (2011). $\triangle F I G$. 6: Mean dynamic ocean topography (MDT) deduced from the difference of the mean sea surface and the GOCE geoid. The mean sea surface is derived from twenty years of satellite radar altimetry. Typical variations of MDT are of the order of 30 centimeters, MDT of the major ocean currents such as Gulf stream, Agulhas, Kuroshio or Antarctic Circumpolar Current reaches one to two meters 